potentially contributing to the observed differential protein release.

Conclusions Hypoxia augments NE release in a PI3K-dependent manner, further increased during COPD exacerbations, and hypoxic neutrophil supernatants injure endothelial cells in vitro. Unbiased characterisation of hypoxic neutrophil secretomes identified several upregulated proteins which may contribute to cellular/tissue damage. In addition to degranulation, NDMP release may underpin differential protein secretion under hypoxia. Hypoxia engenders a neutrophil phenotype with potential to cause local and distant tissue damage in COPD; novel targets in the hypoxic neutrophil secretome may identify new therapeutic opportunities.

\section{S115 MECHANISMS TO REVERSE IMPAIRED MACROPHAGE EFFEROCYTOSIS IN COPD}

${ }^{1}$ EM Ryan, ${ }^{2} \mathrm{R}$ Budd, ${ }^{2} \mathrm{MA}$ Bewley, ${ }^{1} \mathrm{P}$ Coelho, ${ }^{3} \mathrm{~W}$ Rumsey, ${ }^{3} \mathrm{Y}$ Sanchez, ${ }^{1} \mathrm{G}$ Choudhury, ${ }^{1}$ PA Reid, 'DH Dockrell, 'SR Walmsley, ${ }^{1}$ MKB Whyte. 'Department of Respiratory Medicine and MRC Centre for Inflammation Research, University of Edinburgh., Edinburgh, UK; ${ }^{2}$ Department of Infection Immunity and Cardiovascular Disease and The Florey Institute for Host-Pathogen Interactions, University of Sheffield Medical School, Sheffield, UK; ${ }^{3}$ Stress and Repair Discovery Performance Unit, Respiratory Therapy Area, GSK., US

\subsection{6/thoraxjnl-2017-210983.121}

COPD patients have defective innate immunity, characterised in part by macrophage dysfunction. In established disease, both monocyte derived macrophages (MDM) and alveolar macrophages (AM), have impaired phagocytosis of bacteria and apoptotic cells (efferocytosis). Failure to adequately efferocytose dying cells leads to further release of inflammatory mediators and ongoing recruitment of immune cells, culminating in inflammation that is both damaging and ineffective. The transcription factor, Nrf2 is the master regulator of the antioxidant-response-element. Previous work using the non specific Nrf2 agonist, Sulforaphane, has partially restored defective bacterial phagocytosis in COPD AMs, however the specificity and mechanism of action in this context remains unknown. The role of Nrf2 activation in restoring macrophage efferocytosis in COPD has yet to be examined. We questioned if impaired macrophage phagocytosis and efferocytosis in COPD share a common mechanism and consequently if the defect in macrophage efferocytosis can be therapeutically manipulated using highly selective Nrf2 agonists. AMs and MDMs were isolated from patients with established COPD (GOLD stage 1-3). Macrophage efferocytosis of apoptotic neutrophils from each donor was correlated with bacterial internalisation of Streptococcus pneumoniae. Efferocytosis assays were carried out in the presence or absence of Sulforaphane and Compound 7, a highly specific Nrf2 agonist (supplied by GSK). Both COPD MDMs and AMs have significantly impaired phagocytosis of bacteria and apoptotic cells compared to Healthy Controls ( $p$ values all<0.05). Moreover, there was a correlation between donor macrophage phagocytosis of apoptotic cells and of bacteria $(\mathrm{r}=0.71)$. In vitro studies using Sulforaphane enhanced efferocytosis of apoptotic cells in both COPD MDMs and AMs $(\mathrm{p}<0.01)$. This effect was replicated using Compound 7 in both MDMs $(\mathrm{p}=<0.05)$ and AMs. This was more pronounced in current smokers. In summary, we observe a correlation between impaired macrophage phagocytosis and efferocytosis in COPD, suggesting a common mechanism. Furthermore, we describe partial rescue of defective COPD MDM and AM efferocytosis by Sulforaphane and via specific activation of the Nrf2 pathway using Compound 7. Together, these data highlight the importance of the Nrf2 pathway in reversing macrophage dysfunction in COPD patients and provide key mechanistic insights into the underlying defect.

\section{S116 CELL-DISSOCIATED HAEMOPHILUS INFLUENZAE AND BACTERIA-ASSOCIATED INFLAMMATORY MEDIATORS IN THE AIRWAYS OF PATIENTS WITH CHRONIC OBSTRUCTIVE PULMONARY DISEASE}

${ }^{1} \mathrm{SJ}$ Thulborn, ${ }^{2} \mathrm{~A}$ Ceroni, ${ }^{3} \mathrm{~K}$ Haldar, ${ }^{3} \mathrm{~V}$ Mistry, ${ }^{1} \mathrm{~J}$ Cane, ${ }^{3} \mathrm{CE}$ Brightling, ${ }^{3} \mathrm{MR}$ Barer, ${ }^{1} \mathrm{M}$ Bafadhel. ${ }^{1}$ Respiratory Medicine Unit, University Of Oxford, Oxford, UK; ${ }^{2}$ Target Discovery Institute, University Of Oxford, Oxford, UK; ${ }^{3}$ Department of Immunity, Infection and Inflammation, University Of Leicester, Leicester, UK

\subsection{6/thoraxjnl-2017-210983.122}

Background Patients with COPD have a susceptibility to respiratory tract infections associated with increased pulmonary inflammation. Bacteria can reside within the host as cell-associated (attached to host cells via adhesins, pili or biofilm formation) or cell-dissociated bacteria. It is unclear how bacteria-tocell interactions affect pulmonary inflammation and whether

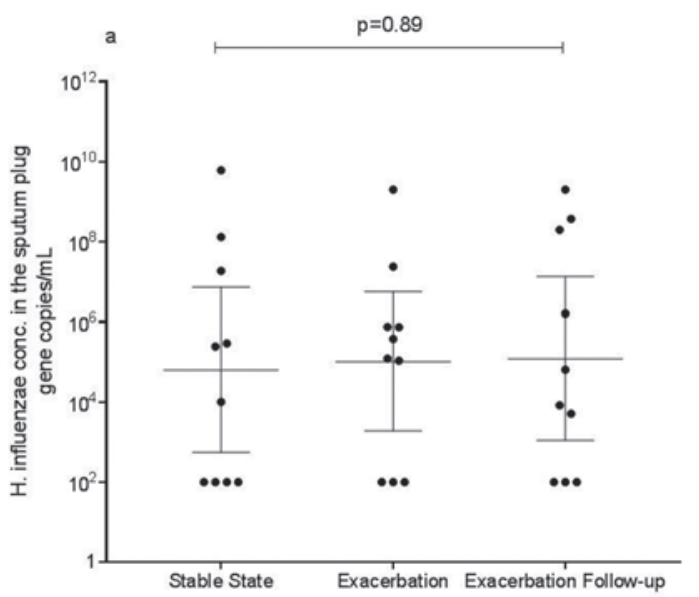

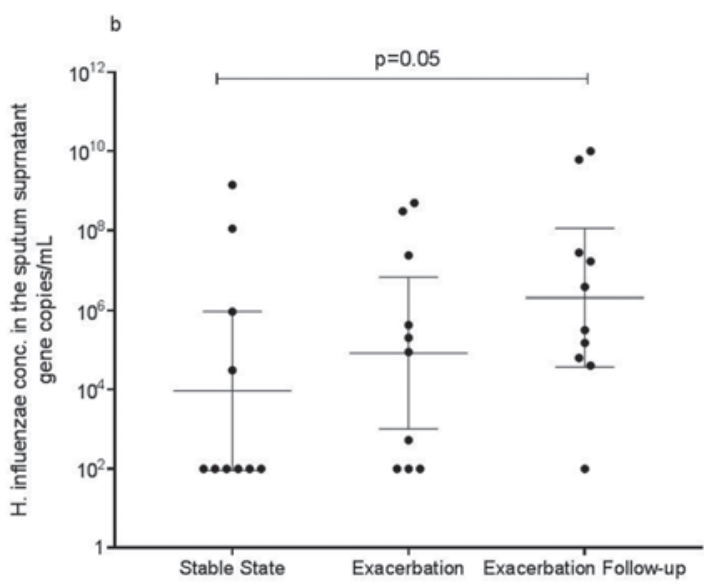

Abstract S116 Figure 1 Levels of $\mathrm{H}$. influenzae in the sputum plug (A) and in the sputum supernatant (B) during an exacerbation time course in 10 paired subjects with COPD. 